\title{
Dihydrocodeine Measurement
}

National Cancer Institute

\section{Source}

National Cancer Institute. Dihydrocodeine Measurement. NCI Thesaurus. Code C74878.

The determination of the amount of dihydrocodeine present in a sample. 